University of Montana

ScholarWorks at University of Montana

7-1-1999

\title{
Evidence of a Solar Origin for Pressure Balance Structures in the High-Latitude Solar Wind
}

\author{
Daniel B. Reisenfeld \\ University of Montana - Missoula, dan.reisenfeld@umontana.edu \\ D. J. McComas
}

J. T. Steinberg

Follow this and additional works at: https://scholarworks.umt.edu/physics_pubs

Part of the Astrophysics and Astronomy Commons

Let us know how access to this document benefits you.

\section{Recommended Citation}

Reisenfeld, Daniel B.; McComas, D. J.; and Steinberg, J. T., "Evidence of a Solar Origin for Pressure Balance Structures in the High-Latitude Solar Wind" (1999). Physics and Astronomy Faculty Publications. 2.

https://scholarworks.umt.edu/physics_pubs/2

This Article is brought to you for free and open access by the Physics and Astronomy at ScholarWorks at University of Montana. It has been accepted for inclusion in Physics and Astronomy Faculty Publications by an authorized administrator of ScholarWorks at University of Montana. For more information, please contact

scholarworks@mso.umt.edu. 


\title{
Evidence of a solar origin for pressure balance structures in the high-latitude solar wind
}

\author{
D. B. Reisenfeld, D. J. McComas, and J. T. Steinberg \\ Los Alamos National Laboratory, Los Alamos, New Mexico
}

\begin{abstract}
Ulysses observations of the high-latitude solar wind have shown that on time scales of $<1$ day, the polar wind is dominated by pressure balance structures (PBSs). Fluctuations of the plasma beta within PBSs appear to be strongly correlated with fluctuations in the helium abundance. The correlation occurs in both the northern and southern hemispheres. In addition, a mechanism is apparently at work in the high-latitude solar wind that dissipates the beta/He correlation over a distance of a few AU. Solar wind composition is established at the base of the corona; thus, the He abundance signature strongly suggests the observed solar wind PBSs are associated with structures low in the solar atmosphere. In particular, high-beta structures appear to originate in locations of enhanced He abundance. We suggest an interpretation of the high-beta portion of PBSs as the solar wind extensions of polar plumes.
\end{abstract}

\section{Introduction}

In situ measurements by the joint ESA/NASA Ulysses mission show that the solar wind at high heliospheric latitudes is remarkably devoid of the large-scale variability and structure commonly observed in the ecliptic plane (McComas et al. 1998 and references therein). The remaining structure is small-scale and falls into three categories: Alfven waves (Smith et al. 1995), compressional structures (McComas et al. 1995) driven by micro-streams of several tens of $\mathrm{km} \mathrm{s}^{-1}$ (Neugebauer et al. 1995), and pressure-balance structures (PBSs), in which fluctuations in the plasma and magnetic pressures balance one another to maintain a comparatively constant total pressure (McComas et al. 1996). These latter authors have shown that on time scales greater than one day, compressional structures dominate the plasma, whereas on time scales less than one day, PBSs dominate.

Remote sensing observations of the high-latitude solar wind close to the Sun show that the large polar coronal holes observed at solar minimum are also relatively structure-free (see, e.g. Krieger et al. 1973). The only significant features are low-contrast, largely radial structures within the coronal holes known as polar plumes. White-light and UV observations indicate that polar plumes are 2-5 times the density of the ambient coronal hole (e.g. Walker et al. 1993). A second distinguishing feature of polar plumes is that they are observed to have anomalous elemental compositions, in particular, a strongly reduced $\mathrm{Ne} / \mathrm{Mg}$ ratio with respect to the ambient solar wind (Wilhelm \& Bodmer 1998). The nature of polar plumes may have considerable bearing on the issue of

Copyright 1999 by the American Geophysical Union.

Paper number 1999GL900368.

0094-8276/99/1999GL900368\$05.00 solar wind acceleration. Plumes have been proposed to play various roles in solar wind dynamics ranging from being unimportant (e.g. Wilhelm et al. 1998) to being the dominant source of high-speed solar wind flow (e.g. Velli et al. 1994).

A number of studies have attempted to find some relationship between Ulysses observations of high-latitude micro-structure and a solar origin, in particular, polar plumes (McComas et al. 1995, Neugebauer et al. 1995, McComas et al. 1996, Poletto et al. 1996, von Steiger et al. 1999). None of these studies found a clear correspondence. The outcome of this apparent disconnect between remote solar and in situ solar wind observations suggests that: 1) the small-scale structure observed by Ulysses in the high-latitude solar wind results from internal evolution of the solar wind, and 2) polar plumes rapidly dissipate into the ambient solar wind, and whatever initial abundance signature they may have is lost.

In this Letter, we present the first clear evidence linking small-scale structure in the polar solar wind to a solar origin. In addition, we suggest an interpretation of these structures as the solar wind extension of polar plumes.

\section{Observations}

Because it is hard to imagine a mechanism that might change particle abundances in the solar wind as it propagates outward, composition signatures are an obvious way to tie solar wind structures far from the Sun to structural elements near the Sun. We therefore searched for associations between the relative abundance of helium in the solar wind (defined as $\mathrm{N}_{\mathrm{He}}{ }^{++} / \mathrm{N}_{\mathrm{H}^{+}}$and denoted hereafter as [He]), and other plasma properties. Ulysses observations of $[\mathrm{He}]$ show that at high latitudes, $[\mathrm{He}]$ varies by only \pm 15 percent (at $1 \sigma$ ) about a value of 0.044 (Barraclough et al. 1996). This is quite small compared to $[\mathrm{He}]$ variations observed at low latitudes, suggesting that any effect will be subtle at best. Neugebauer et. al. (1995) examined [He] with respect to micro-streams and found a systematic variation in the alpha-proton flux ratio. However, the evidence was not sufficiently compelling for them to tie micro-streams to a solar origin. Von Steiger et al. (1999) also searched for compositional variations within micro-streams and found no clear association.

In McComas et al. (1996) the authors approached the question of whether PBSs were of solar origin by examining the global average properties of PBSs, including [He], and comparing these to the undifferentiated solar wind. They found no significant differences. However, this does not preclude the possibility that there are structures within PBSs having a solar origin. In fact McComas et al. found strong correlations between fluctuations in [He] and the plasma beta, $\beta$, within PBSs for a few case intervals. Though they warned that these correlations can be misleading when only a small number of cases are considered, they suggested the matter 
requires closer analysis. Following on this previous work, we parameterize PBSs by use of $\beta$, which measures the relative pressures of the plasma and magnetic field. We then perform a systematic analysis of the correlation between [He] and $\beta$ within the PBSs of the high-latitude solar wind in an attempt to identify a solar origin for these structures.

This study incorporates Ulysses solar wind plasma observations from the Los Alamos solar wind ion and electron spectrometers (Bame et al. 1992) and magnetic field observations from the Imperial College/JPL magnetometer (Balough et al. 1992). The plasma moments have been calculated from integration of the particle distribution functions, providing independent parameters for the proton, $\alpha$-particle, core electron, and halo electron pressure components. The one exception is that the electron core density has been determined from the other three component densities under the assumption of charge neutrality. Determining the core density in this way removes the uncertainty due to the large fraction of core electrons having energies below the typical spacecraft potential of several $\mathrm{eV}$.

The plasma measurements are combined to give the He abundance and the total plasma pressure. The plasma pressure is then divided by the magnetic pressure, $P_{m}=B^{2} / 2 \mu_{0}$ to give $\beta$. In this study we use one-hour averaged values of the plasma parameters and magnetic field data, extending over almost the entire first Ulysses solar polar orbit from April 1994 to February 1996. During this period the spacecraft was continuously immersed in high-speed flow with the exception of February and March 1995, when Ulysses underwent the fast latitude scan around perihelion.

In Fig. la we show the evolution of $[\mathrm{He}]$ and $\beta$ for a sevenday interval when Ulysses neared its maximum heliospheric latitude of $80^{\circ} \mathrm{N}$. To emphasize the relationship between [He] and PBSs, we have high-pass filtered the data in the frequency domain (McComas et al. 1996). We choose a cutoff of 12 hours which effectively removes compressional features. A significant correlation is often present in the temporal/spatial variation of [He] and $\beta$. To quantify the correlation, we have calculated the correlation coefficient between [He] and $\beta$ within a sliding 12 -hour window centered on consecutive one-hour samples. The result is shown in Fig.

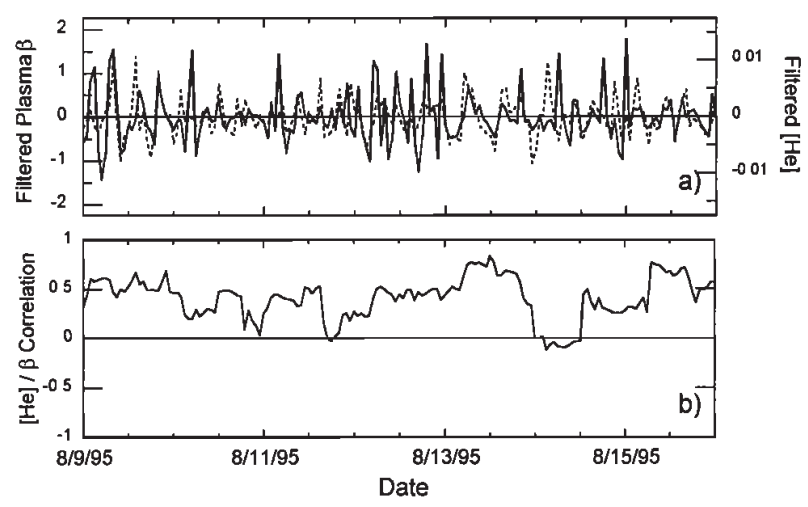

Figure 1. a) Overlay of $\beta$ (solid line) and [He] (dashed line) as a function of time for seven consecutive days during the Ulysses northern polar passage. The observed correlation is quantified in $b$ ), which plots the running correlation coefficient between $\beta$ and [He].

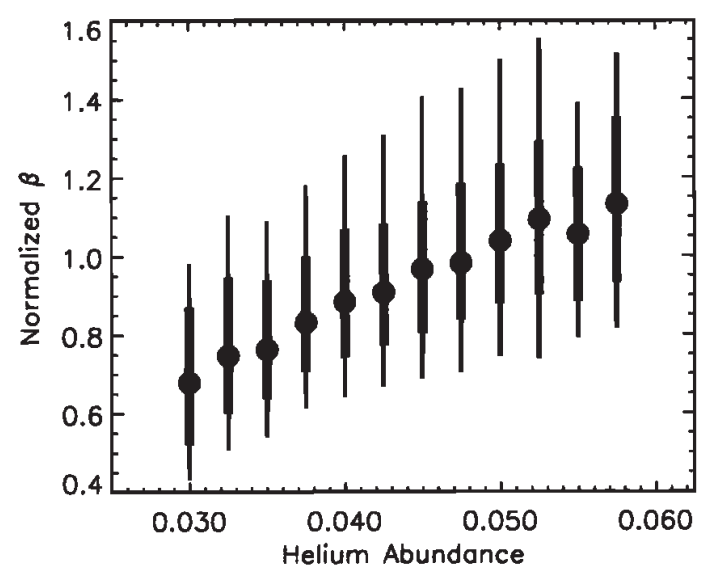

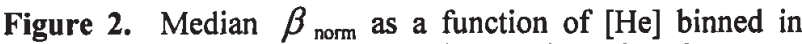
intervals of 0.0025 for those times when the short-term fluctuations in $\beta$ and [He] are correlated $(r>0.3)$. The vertical bars indicate the shape of the distribution within each bin. The thin vertical bars span the 10- and 90-percentiles, and the thick vertical bars span the $25-$ and 75 -percentiles.

1b. A positive correlation is present over almost the entire interval, at times reaching as high as 0.8 , with an average correlation of 0.4 . The net positive correlation shown here persists throughout both Ulysses northern and southern polar passages.

The correspondence between variations in [He] and $\beta$ suggests that at those times when a significant correlation is present, a correlation may also exist between the magnitudes of [He] and $\beta$. Figure 2 plots $\beta$ vs. [He] for those times when the 12-hour running correlation described above is $>0.3$ and when Ulysses was poleward of $45^{\circ} \mathrm{N}$. Beta is not a conserved quantity, but rather shows a weak dependence on distance from the Sun. To remove the radial dependence we define a new parameter, $\beta_{\text {norm, }}$ which is determined by normalizing the values of $\beta$ in consecutive two-week intervals to the mean in each respective interval. Thus, the re-scaled values $\beta_{\text {norm }}$ are centered on 1 .

Figure 2 shows a histogram of the median $\beta_{\text {norm }}$ vs. [He]. Clearly, a statistically significant dependence exists between $\beta$ and [He]: the median $\beta_{\text {norm }}$ smoothly grows from 0.67 to 1.15 (an increase of $70 \%$ ), a trend that is also reflected by smooth variation of the percentile values. Note the $\beta_{\text {norm }} /[\mathrm{He}]$ relation persists across a factor of two span in [He], the entire range over which [He] varies in the high latitude solar wind.

Numerous tests were performed to verify that the most appropriate comparison is between [He] and $\beta$, and not between $[\mathrm{He}]$ and any other plasma parameter, in particular, one of the constituents of $\beta$. Figure 3 is similar to Fig. 2, except that in addition to comparing the total plasma $\beta$ to [He], the normalized $\beta$ components (protons, alphas, core and halo electrons) are also binned according to [He]. As in Fig. 2 , the total $\beta$ and its components are normalized to remove the radial dependence. During this period, the actual contributions of each plasma components to the total $\beta$ was on average $55 \%$ for the protons, $9 \%$ for the alphas, $19 \%$ for the core electrons, and $15 \%$ for the halo electrons.

The most striking aspect of Fig. 3 is that the dependence of individual $\beta$ components on $[\mathrm{He}]$ is remarkably consistent. Closer examination of the detailed dependencies (not presented here) shows that the variation of the magnetic 


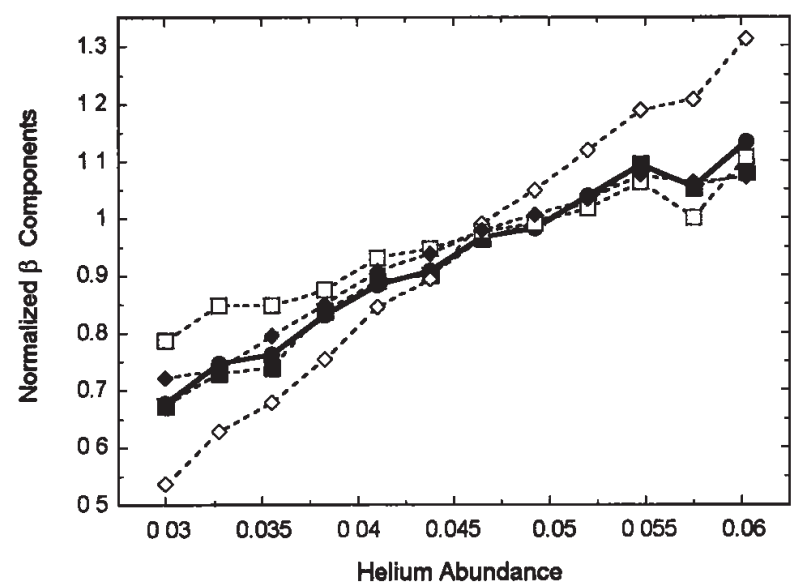

Figure 3. Median $\beta_{\text {norm }}$ vs. [He] calculated using the total (solid circles) and individual component pressures: proton (solid squares), $\alpha$-particle (open diamonds), core electron (open squares), and halo electron (solid diamonds).

pressure with [He] dominates, which explains the consistency across $\beta$ components since $P_{m}$ is common to all. The [He] dependence of $P_{m}$ accounts for about two-thirds of the $\beta$ variation, while the four plasma pressure components account for the remaining third. Of these, the variation of the proton pressure makes the most significant contribution, as it is the largest pressure component. Although the $\alpha$-particle pressure $\left(P_{\alpha}\right)$ exhibits the strongest correlation with [He] (reflected in the steep slope of the $\alpha$-particle $\beta_{\text {norm }}$ curve in Fig. 3 ), this correlation does not contribute significantly to the overall trend since $P_{\alpha}$ makes up only $9 \%$ of the total pressure. Furthermore, the strong $P_{\alpha}$ dependence is ultimately unimportant because it includes a false correlation: $P_{\alpha}$ and [He] are not independent parameters as they both are linearly dependent on the $\alpha$-particle density. Finally, we note that the data shown in Fig. 3 essentially rule out the possibility that the $[\mathrm{He}] / \beta$ correlation is an instrument effect since the plasma

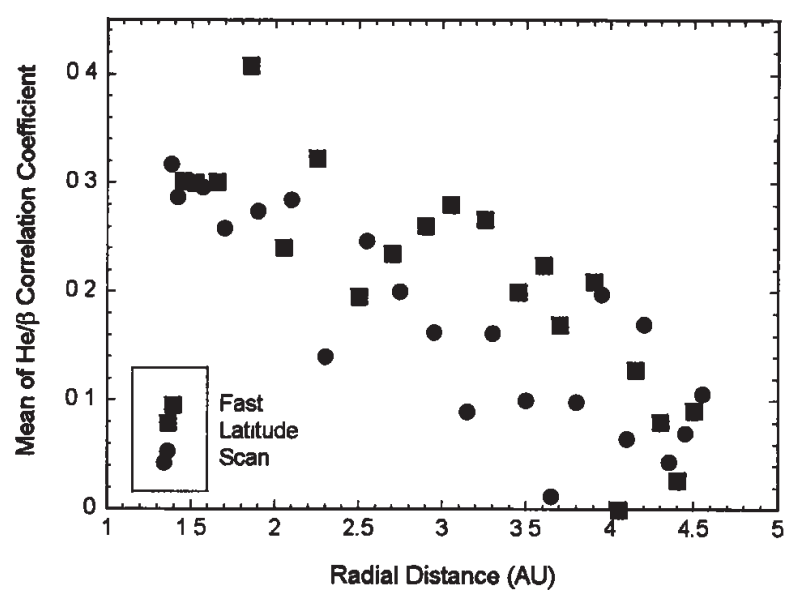

Figure 4. Correlation of [He] and $\beta$ temporal fluctuations as a function of spacecraft distance from the Sun. Plotted is the $[\mathrm{He}] / \beta$ correlation coefficient (described in Fig. 1b) averaged over 1-month intervals (except for the points within 1.5 AU, which are averaged over 2-week intervals). Circles indicate the northern polar pass, and squares indicate the southern.

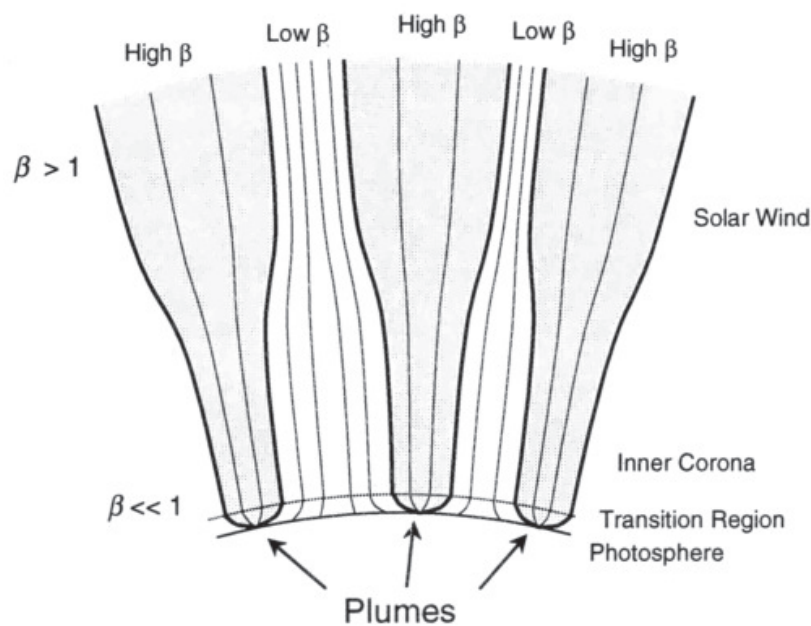

Figure 5. Illustration of a possible scenario associating high$\beta$ structures with the extension of polar plumes into the solar wind.

and field measurements are made by three different instruments and are therefore completely independent.

The fact that $\beta$ and its components are correlated with [He] strongly indicates that the pressure balance structures are formed near the base of the corona, the location where the He abundance is established. Additional evidence that the PBSs originate near the Sun is shown in Fig. 4, where the degree of $[\mathrm{He}] / \beta$ correlation is plotted as a function heliographic distance. Each point represents the mean of the 12-hour running correlation (see Fig. 1b) for a one-month period, plotted at the average radial distance of the spacecraft for that month. A clear radial trend is indicated. The mean correlation drops from $\sim 0.3$ at $1.5 \mathrm{AU}$ to $\sim 0.05$ at $4.5 \mathrm{AU}$, indicating that the coherence between [He] and $\beta$ decays as the solar wind evolves from the Sun. Data for both the northern and southern passes are shown and are in agreement.

The four points at the smallest radial distance are exceptions to this trend. They occurred during the period of the Ulysses rapid latitude scan when the spacecraft was within $25^{\circ}$ of the heliospheric equator, and therefore within the solar wind extension of the streamer belt. This then suggests that whatever mechanism establishes the $[\mathrm{He}] / \beta$ correlation is either absent in the streamer belt, or if it is present, the coherence dissipates much nearer the Sun.

\section{Discussion: Association with Polar Plumes}

To summarize the observations, variations in the solar wind $\mathrm{He}$ abundance are often well correlated with variations in $\beta$; the correlation diminishes with radial distance; and the correlation is absent at low latitudes. We take this as strong evidence that many of the pressure balance structures in the solar wind are of a solar origin, and they most likely originate at high solar latitudes.

Associating the PBSs with polar plumes is more tentative. As mentioned in the Introduction, not all researchers agree that polar plumes extend into the solar wind, in which case our observations may be the manifestation of structures other than plumes (see, e.g., Wilhelm et al. 1998). However, since polar plumes are the only observed quasi-stationary high- 
latitude coronal structures (during solar minimum), it is hard to imagine where else high-latitude solar wind structure can originate. Plumes are believed to originate at the boundaries of chromospheric networks where magnetic flux is concentrated through supergranular convection (Berger \& Title 1996). In the transition region $(\sim 7000 \mathrm{~km}$ above the photosphere), the magnetic field inside plumes is $\sim 10-100$ $\mathrm{G}$, whereas outside plumes, the field is $\sim 5-10 \mathrm{G}$ (DeForest et al. 1997). Both inside and outside the plumes, $\beta \ll 1$, so the plasma dynamics are completely dominated by the magnetic field. Suess et al. (1998) applied a straightforward potential field model to show that plumes rapidly expand above the transition region, and by a height of $\sim 35,000 \mathrm{~km}$, the plumeinterplume regions are in a state of pressure balance, of nearly equal field strength. At this height, what distinguishes plumes from the ambient coronal hole is the factor of 2 to 5 higher density inside plumes (Walker et al. 1993). Temperature differences are also present but they are small, the ion temperature being at most $30 \%$ lower within plumes (DeForest et al. 1997). Thus the ratio of plume to interplume $\beta$ is in the range 1.3 to 3.5 .

Figure 5 depicts a scenario describing how PBSs in the solar wind may be associated with polar plumes. As the solar wind propagates outward, the plumes expand super-radially, compressing the interplume material. This is due to the more rapid drop in magnetic pressure (which varies as $\sim R^{-4}$ near the Sun) than plasma pressure (which varies as $\sim R^{-2.5}-R^{-3}$ ). Thus at some distance, $\beta$ becomes $>1$ and the plasma pressure dominates the magnetic pressure in governing plasma dynamics. Because of their higher density, the plumes expand to maintain pressure balance. Regardless of how $\beta$ subsequently evolves with distance, so long as material does not mix across field lines, $\beta$ within plumes will always remain higher than in adjoining interplume regions.

In support of the notion that PBSs in the high latitude solar wind are of solar origin is the observed enrichment of $\mathrm{He}$ in the high- $\beta$ regions. Abundance anomalies do appear in solar observations of plumes (Wilhelm \& Bodmer 1998). Unfortunately, no abundance measurements exist as yet having sufficient resolution to distinguish plume from interplume [He]. We are then left to speculate on what mechanisms might enrich $\mathrm{He}$ at the base of the corona. Perhaps the He inside plumes is more efficiently coupled to the accelerating solar wind through a higher Coulomb collision rate- a consequence of the enhanced plume density. This would yield a positive correlation between $[\mathrm{He}]$ and $\beta$ in interplanetary space, just as we observe.

The observed dissipation of the high-latitude $[\mathrm{He}] / \beta$ correlation with distance also supports our plume scenario. Some solar wind models predict the disruption of plumes as they propagate outward. For example, the model of Suess (1998), postulates velocity shear between plumes and the ambient solar wind lead to turbulent disruption once the velocity difference exceeds the local Alfven speed (KelvinHelmholtz instability). Alternatively, plume dissipation may be a consequence of the dominance of compressional events at greater heliospheric distances (Velli et al. 1994). This may also explain the absence of a $[\mathrm{He}] / \beta$ correlation at low latitudes, where compressional events are more prevalent.

In summary, we conclude that contrary to earlier studies, there is clear evidence that the high latitude solar wind beyond $1 \mathrm{AU}$ still carries information about the structure of the inner corona. In particular, high- $\beta$ structures within PBSs in the solar wind appear to originate in locations of enhanced He abundance. These locations may correspond to the bases of polar plumes. There is also evidence that a mechanism is at work in the evolving high-latitude solar wind that dissipates the abundance signature on a distance scale of a few AU. Clearly much more work needs to be done to complete our understanding of the origin and evolution of solar wind PBSs. Further studies are planned to investigate the properties of individual high- $\beta$ structures, and to search for correlations with other solar wind elemental abundances.

Acknowledgements. We thank Ed Santiago for invaluable assistance with SWOOPS data reduction. This work was carried out under the auspicies of the United States Department of Energy with support from the NASA Ulysses program.

\section{References}

Balogh, A., et al., The magnetic field investigation on the Ulysses mission: Instrumentation and preliminary scientific results, Astron. And Astrophys., Suppl. Ser., 92, 221, 1992.

Bame, S.J., et al., The Ulysses solar wind plasma experiment, Astron. And Astrophys., Suppl. Ser., 92, 237, 1992.

Barraclough, B.L., et al., He abundance variations in the solar wind: Observations from Ulysses, Solar Wind 8, AIP Proc. 382, Ed. D. Winterhalter, et. al., 277, 1996.

Berger, T.E. \& Title, A.M., On the dynamics of small scale solar magnetic elements, Astrophys. J., 463, 365 .

DeForest, C.E., et al., Polar plume anatomy: Results of a coordinated observation, Solar Phys., 175, 393, 1997

Krieger, A. S., et al., A coronal hole and its identification as the source of a high velocity solar wind stream, Solar Phys., 29, 505, 1973.

McComas, D.J., et al., Structures in the polar solar wind: Plasma and field observations from Ulysses, J. Geophys. Res., 100, 19,893, 1995.

McComas, D.J., et al., Ulysses observations of pressure-balance structures in the polar solar wind, Astron. and Astrophys., 316, $368,1996$.

McComas, D.J., et al., Ulysses return to the slow solar wind, Geophys. Res. Lett.,25, 1, 1998.

Neugebauer, M., et al., Ulysses observations of microstreams in the solar wind from coronal holes, J. Geophys. Res., 100, 23,389, 1995.

Poletto, G., et al., Searching for coronal plumes in Ulysses observations of the far solar wind, Astro. and Astrophys., 316, $374,1996$.

Smith, E.J., et al., Ulysses observations of Alfven waves in the southern and northern solar hemispheres, Geophys. Res. Lett., 22, 3381, 1995.

Suess, S.T., Models of plumes: Their flow, their geometric spreading, and their mixing with interplume flow, Solar Jets and Coronal Plumes, ESA SP-421, 223, 1998.

Suess, S.T., et al., The geometric spreading of coronal plumes and coronal holes, Solar Phys., 180, 231, 1998.

Velli, M., et al., Coronal plumes and fine scale structure in high speed solar wind streams, Space Sci. Rev., 70, 391, 1994.

von Steiger, R., et al., Composition variations in fast solar wind streams, Solar Wind 9, AIP Proc. 471, Ed. S.R. Habbal, in print, 1999.

Walker, A.B.C., et al., Thermal and density structure of polar plumes, Solar Phys., 148, 239, 1993.

Wilhelm, K., et al., The solar corona above polar coronal holes as seen by SUMER on SOHO, Astrophys. J., 500, 1023, 1998.

Wilhelm, K. \& Bodmer, R., Solar EUV and UV emission line observations above a polar coronal hole, Space Sci. Rev., 85, 371, 1998.

D. J. McComas, D. B. Reisenfeld, and J. T. Steinberg, M/S-D44, Los Alamos National Laboratory, Los Alamos, NM 87545, USA (email: dreisen@lanl.gov)

(Received March 5, 1999; revised April 27, 1999; accepted May 3, 1999.) 